\title{
MACHINE LEARNING IN AGRICULTURE: TECHNIQUES AND APPLICATIONS
}

\author{
Neha Yadav \\ Department of CSE \\ Lovely Professional University, \\ Phagwara, Punjab, India
}

\author{
Sk Md Alfayeed \\ Department of CSE \\ Lovely Professional University, \\ Phagwara, Punjab, India
}

\author{
Ankita Wadhawan \\ Department of CSE \\ Lovely Professional University, \\ Phagwara, Punjab, India
}

\begin{abstract}
Through Big Data and High-Performance Computing, Machine Learning has evolved, creating new possibilities for data-intensive research in the area of agriculture. For precision analysis, numerous computing methods, such as and neural networks, $k$-means etc. have been used in the past. The following techniques: Artificial Neural Network, Information Fuzzy, Support Vector Machines, Decision Tree, Bayesian Belief Network, Regression Analysis, etc. are the most commonly used methods. This paper offers an extensive review of work on machine learning applications in agricultural field.
\end{abstract}

Keywords - Machine Learning, SVM, ANN, Image Processing, K-Means, ANN.

\section{INTRODUCTION}

Civilization was born in agriculture. India, a country based mainly on agricultural productivity, is an agricultural state and its economy. Agriculture is, therefore, the foundation of every industry in India. In manufacturing, agriculture, and related industries such as forestry and fishing, India is now ranked second globally, expected to account for 14.5 per cent of GDP in 2015, and about 50 per cent of total labor. India's GDP improves agriculture economically, and this presents threats and also impacts the stable food output.

Environmental considerations mainly influence agriculture. Climate affects crop development and growth, causing significant variation in intra-seasonal yields. Furthermore, spatial variability in soil effects causes spatial variability in yield by interacting with the weather. Planting, fertilizers, drainage, lying, etc. in agricultural crops may be used to mitigate lack of yield due to weather impact. This is a vital tool for the optimization of crops and the evaluation of crop area insurance contracts.

G Yamuna describes changes climatically, occasionally, as a result of pollution, population, the handling of solid waste, surface and water hydrology, etc. and climate change impacts on developing worlds. It helps us to get the impression that we must always examine climate change production. [11] The analysis described rainfall-runoff using the artificial neural network and concluded that the artificial neural network models accurately predict the data.[12] The water level of the reservoir via the neural network is expected.[13]
Machine learning means giving machine information. Computer teaching techniques, including controlled and unattended instruction, are unique. Supervised learning ensures a teacher who guides the curriculum with teaching experiences and can find the exact interpretation of new information. Many supervised learning definitions include an artificial neural network, Bayesian network, decision tree, vectors, ID3, knearest neighbor, and the secret Markov model. Unsupervised machine learning allows the system a large amount of data and reveals how they function and their relationships. Yet unrestricted testing can be used to discover hidden patterns in the tests. Several definitions of unsupervised learning algorithms are the nearest neighbor, the self-organizing diagram, partial clustering, hierarchy, k-means clustering, and so on.

\section{Classification Of MACHINE LEARNING}

\section{A. Supervised Learning}

Label data are used to train the machine in supervised learning, meaning that certain data are already marked with the attribute names. It is comparable with learning in the presence of a professor or supervisor.

\section{$B$. Unsupervised Learning}

Unsupervised learning is a method for machine learning that you don't have to supervise the pattern. Rather, you must encourage the model to learn knowledge on its own using unlabeled data.

Algorithms of unsupervised learning enable you to carry out more complicated computing tasks than supervised learning. Unsupervised with other natural learning methods, uncontrolled learning may be more unpredictable.

\section{Reinforcement Learning}

Reinforcement learning is a conditioning model machine learning to render a series of choices. In an unpredictable, possibly complex climate, the agent learns to pursue a target. A game-like scenario confronts artificial intelligence in reinforcement learning. Here the machine uses trial and error approach. 


\section{International Journal of Engineering Applied Sciences and Technology, 2020 \\ Vol. 5, Issue 7, ISSN No. 2455-2143, Pages 118-122 \\ Published Online November 2020 in IJEAST (http://www.ijeast.com)}

\section{LEARNING MODELS OF ML}

\section{A. Regression}

Regression represents a controlled learning model that aims to predict an output variable in accordance with the known input variables. Linear regression and functional regression [17], as well as progressive regression [18], are the most popular algorithms of this kind. More complicated regression algorithms such as ordinary mean squares regression, multivariate adaptive regression splines, multiple linear regression, cubistic, and locally calculated smoothing for scatterplots were also created.

\section{B. Clustering}

The process of identifying elements that are close to each other and distinct from entities in the other classes is clustering or clustering of clusters study - primarily used to analyze data. In many industries, clusters are used for machine learning, pattern recognition, processing of images, recovery of knowledge, and farming. There really are various clustering algorithms like kmeans, k-medoids, and so on, but k-means are the most effective clustering algorithm.

\section{Bayesian Models}

The Bayesian models are the probabilistic class of graphic models in which an experiment is carried out within the Bayesian reference sense. This model is in the supervised category and can be used to solve problems of both classification or regression. The most prominent algorithms of the literature include naive bays [19], naive bays, multi-intellectual naive bays, Bayesian network, Gaussian mixture, and Bayesian creed network [20].

\section{Instance-Based Models}

The Models based on instances, which learn by connection with examples in the training set, are memory models. They create theories from the data available explicitly without holding a variety of abstractions and using only specific instances; they produce classification or regression projections. The drawback of such models is that data increases their complexity. Locally weighted learning and learning vector scale quantization are the most common algorithms in this category.

\section{E. Decision Trees}

The Decision tree is the structure of a tree-like system of classification or regression. It slowly organizes the dataset into smaller homogenous subsets (subpopulations), thus creating a tree graph. Each tree structure internal node represents a different comparison on a given function in pairs, whereas each branch represents the result. The leaf nodes reflect the final decision and prediction after the root-to-leaf direction (expressed as a classification rule).

\section{F. Supervised Learning}

Reinforcement learning is a conditioning model machine learning to render a series of choices. In an unpredictable, possibly complex climate, the agent learns to pursue a target. A game-like scenario confronts artificial intelligence in reinforcement learning. Here the machine uses trial and error approach.

\section{TeChniques And Methodology}

\section{A. Information Fuzzy Network}

The IFNs are a greedy machine algorithm for supervised education-information fudging networks (IFN). Info Fuzzy Network is also regarded as the data structure generated by the learning algorithm. The building of the IFN is close to the building of decision trees. Nonetheless, IFN builds a graph and does not construct a circle.

\section{B. Artificial Neural Networks}

The network of human-like neurons is the Artificial Neural Network. It builds on the biological processes of the human brain. This is one example of supervised education. A neural network must be educated once; similar patterns can be expected in future data; for example, if the input data is incorrect or incomplete, practical solutions to problems can be sought. By the addition of more and more data, ANN's accuracy continues to increase. ANNs are also capable, without knowing the underlying principles of its complexity. ANNs can extract inputoutput relationships for every method.

\section{Support Vector Machines}

The study of time series is a technique for measurement of time on parametric, linear data in order to derive meaningful statistics and other data characteristics. Forecast time series is a guide for predicting future values based on values previously observed. Time series analysis methods, like frequency field and time field, parametric or non-parametric, are available for both linear and non-linear, univariate, and multivariate analysis. The important tool for crop forecasting is time series analysis. In the function of time, we can see the connection between yield and time by taking the dependent variable yield.

\section{Bayesian Belief Network}

SVM constructs a linear, separating hyperplane for classifying data instances, intrinsically a binary classifier. By turn the initial function space into a higher dimensional feature space, the classification capabilities of modern SVMs will significantly be improved by using the kernel trick. For grouping, regression, and clustering, SVMs were used. SVM addresses the overfit problems, which occur in high-dimensional spaces, on the basis of global optimization, and are attractive in various applications. The most popular SVM algorithms are supporting a vector regression, a vector machine supporting smaller squares, and a successive vector machine supporting a projection algorithm [8].

\section{ApPlicAtions Of Ml In Agriculture}




\section{International Journal of Engineering Applied Sciences and Technology, 2020 \\ Vol. 5, Issue 7, ISSN No. 2455-2143, Pages 118-122 \\ Published Online November 2020 in IJEAST (http://www.ijeast.com)}

\section{A. Species Management}

\section{Breeding of Species}

The selection of a plant is a tedious process in which the quality of water, dietary resources, tolerance to climate change, resistance to disease, and the value of nutrients or a better taste need to be studied. In order to evaluate crop production, the machine learning, in particular the deep learning algorithms can be useful for better activity logging.

\section{Recognition of Species}

Although a human expert can recognize the species by seeing, machines also can achieve better and quicker results by studying the Morphology of the leaf vein with more knowledge about the Leaf properties. The grouping of leaves is based on color and shape.

\section{B. Field Conditions Management}

\section{Management of Soil}

Soil is a heterogeneous natural resource with complex processes and vague mechanisms for farming specialists. The temperature alone can offer clues into the impacts on the global development of climate change. In order to understand the complexities of vegetation and impingement to agriculture, deep learning algorithms study evaporation cycles, soil humidity, and temperature.

\section{Management of Water}

The hydrological, ecosystem and agronomic equilibrium are influenced by the water management of agriculture. Using more effective use of irrigation systems and forecasting daytime dew point temperature, the most advanced applications to date are correlated with an estimation of regular, weekly or monthly evapotranspiration which helps to recognize predicted weather phenomenon and to assess evapotranspiration and evaporation.

\section{Crop Management}

\section{Prediction of Yield}

Precision farming has a yield forecast that is one of the most important and popular subjects because it defines yield mapping and estimation, matching demand with the crop offer and farm management. Moderate methods have gone much further than the basic, historical prediction, but have integrated computer vision techniques, which provide details on the way and a detailed, multi-dimensional agricultural, meteorological and economic study that maximizes farmers and the population's yields.

\section{Quality of Crop}

Precise identification and assessment of crop quality will increase the price of the product and decrease waste. Machines can use obviously meaningless data and links in conjunction with human experts to discover and identify new characteristics that are part of the overall nature of crops.

\section{Detection of Disease}

Uniform spray of pesticides across the crop area, both in openair conditions and in greenhouse conditions, is the most common practice in pest and disease control. This strategy allows for massive quantities of pesticides to be successful, resulting in major financial and environmental costs. In terms of time, location and impacted species, ML is used as part of the overall precision agricultural management system.

\section{Detection of Weed}

Weeds are the main threats to crop production apart from diseases. The greatest problem in the battle against weeds is that crops are hard to detect and discriminate against. The identification and filtering of cannabis at low cost and without pollution and side effects enhances computer vision and ML algorithms. In the future, such developments will lead to the destruction of weeds by robotics that reduces the need for herbicides.

\section{Livestock Management}

\section{Production of Livestock Disease}

Machine learning provides precise prediction and estimation of agricultural parameters similar to crop production, with a view to maximizing the economic efficiency of systems such as animal husbandry and livestock production. In order to allow agriculturists to modify their diets and conditions, for example, weight predicting systems can estimate future weights 150 days in advance.

\section{RELATED WORKS}

Yethiraj et al. (2012) examined data mining applications and found that there are several algorithms and techniques used in agriculture.

Babu (2013) addressed the criteria and the strategy required to develop a precision-based farming software model. This analyzes the fundamental principles of precision agriculture extensively. They started from the fundamental principles of precision agriculture and worked towards the creation of a model to sustain it. This paper presents a model applying the concepts of precision agriculture to small and open farms at the level of individual farmers and crops, which cause a degree of variability regulation.

Nasirahmadi and Behroozi-Khazaei (2013) used machinelearning techniques on colored harvests in his paper to identify ten local Iranian beans. Three successive steps were taken to identify the varieties of bovine beans.

Dahikar and Rode et al. (2014) proposed a model to predict the right fertilizer by using the artificial neural network and suggested an appropriate crop dependent on the soil's characteristics for crop yield.

Shi et al. (2015) discussed the advantages of the Internet of Things (IoT) in agriculture. The quality and quantity of the agricultural product are greatly reduced in plant diseases and insect plagues so that forecasting of plant diseases and insectbased pests is important and very critical. The principal goal is to accomplish IoT knowledge and compilation by the use of machine learning algorithms. 


\section{International Journal of Engineering Applied Sciences and Technology, 2020 \\ Vol. 5, Issue 7, ISSN No. 2455-2143, Pages 118-122 \\ Published Online November 2020 in IJEAST (http://www.ijeast.com)}

Savla et al. (2015) provided a comparative analysis regarding classification algorithms and of their success in precision agriculture yield prediction. Such algorithms are used to estimate the performance of soybean plants for a number of years in a data set. The predictive yield algorithms used in this article are Vectors, Random Forest, Neural Networks, REP Tree, Bagging and Bayes help. Eventually, bagging is the best predictor among the aforementioned algorithms because the bagging misalignment is the least with a mean absolute error of 18985. The conclusion is that bagging is the best predictor.

Manjula et al. (2015) pointed out the importance of forecasting crop yields and its relation to the strategic agricultural policy of a country. An Extensible Architecture of Crop Yield Prediction is established (XCYPF). It makes it easier for different methods to be included in the estimation of crop yield. A method to help people forecast crop yields for different crops with depending and independent variables were also created.

Mishra et al. (2016) have done research studies on the importance of machine learning techniques within agriculture development will be reassessed.

Zhao et al. (2017) addressed a short wind speed model for casting, which includes a new active learning algorithm to pick samples. For the sample search, active learning is used for machine learning. Active learning was beneficial for applications with a large number of research examples for estimating wind speed.

Akshatha and Shreedhara (2018) proposed A recommendation system by a group model with majority voting technique using the Random Tree, K-Nearest Neighbor and Naive Bays as students in order to recommend a plant with high precision and efficiency for specific site parameters.

Chandgude et al. (2018) discussed the idea of a crop surveillance system. Different methods of machine learning relate to environmentally sensed data through sensors.

Mohiuddin \& Alam (2019) discussed the introduction of modern technology, in particular, artificial agricultural intelligence to improve the system of food production, establish a subtle ecosystem for crops, and modify plantation to generate more less-resourced crops.

\section{CONCLUSION}

Currently, there is an increasing number of applications of machine learning approach available in agriculture field that can be examined using a large amount of currently available data from various platforms. It is a sophisticated area of research, and it will be growing in the future. The development of an effective model will have some benefits with respect to the conventional forecast system. In this paper, we discussed various approaches and applications of machine learning in agriculture. In the future, we would like to extend our work by the implementation of some of the approaches to propose an advance approach.

\section{ACKNOWLEDGEMENT}

We would like to take the opportunity to acknowledge the support and help of all who have assisted us in the research. Without their contribution and advice, we would have never been able to progress with the work in the research. Firstly, we would like to sincerely acknowledge my research mentor Ms. Ankita Wadhawan, for her guidance, support, technical knowledge and encouragement in the whole research process and work. The information and feedback provided were extremely helpful and useful for this paper.

\section{REFERENCE}

[1] Truong, T., Dinh, A., \& Wahid, K. (2017, April). An IoT environmental data collection system for fungal detection in crop fields. In 2017 IEEE 30th Canadian Conference on Electrical and Computer Engineering (CCECE) (pp. 1-4). IEEE.

[2] Shi, Y., Wang, Z., Wang, X., \& Zhang, S. (2015, May). Internet of things application to monitoring plant disease and insect pests. In 2015 International conference on Applied Science and Engineering Innovation. Atlantis Press.

[3] Zhao, X., Wei, H., Zhang, C., \& Zhang, K. (2017, May). Selective sampling using active learning for short-term wind speed prediction. In 2017 29th Chinese Control And Decision Conference (CCDC) (pp. 6717-6720). IEEE.

[4] Vamanan, R., \& Ramar, K. (2011). Classification of agricultural land soils a data mining approach. International Journal on Computer Science and Engineering, ISSN, 0975-3397.

[5] Yethiraj, N. G. (2012). Applying data mining techniques in the field of agriculture and allied sciences. International Journal of Business Intelligents ISSN, 2278-2400.

[6] Rehman, T. U., Mahmud, M. S., Chang, Y. K., Jin, J., \& Shin, J. (2019). Current and future applications of statistical machine learning algorithms for agricultural machine vision systems. Computers and electronics in agriculture, 156, 585-605.

[7] Mohiuddin, K. \& Alam, M. M.. (2019) A short review on agriculture based on machine learning and image processing. Acta scientific agriculture 3.5, 55-59.

[8] Mishra, S., Mishra, D., \& Santra, G. H. (2016). Applications of machine learning techniques in agricultural crop production: a review paper. Indian J. Sci. Technol, 9(38), 1-14.

[9] Akshatha, K. R., \& Shreedhara, K. S. (2018). Implementation of machine learning algorithms for crop recommendation using precision agriculture. International 


\section{International Journal of Engineering Applied Sciences and Technology, 2020 \\ Vol. 5, Issue 7, ISSN No. 2455-2143, Pages 118-122 \\ Published Online November 2020 in IJEAST (http://www.ijeast.com)}

Journal of Research in Engineering, Science and Management (IJRESM), 1(6), 58-60.

[10] Chandgude, A., Harpale, N., Jadhav, D., Pawar, P., \& Patil, S. M. (2018). A Review on Machine Learning Algorithm Used For Crop Monitoring System in Agriculture. International Research Journal of Engineering and Technology (IRJET), 5(04), 1470.

[11] Yamuna, G.. (2016). Impacts of Climate Change in the Developing World. Indian Journal of Science and Technology. 9. 10.17485/ijst/2016/v9i6/87668.

[12] Chakravarti, A., Joshi, N., \& Panjiar, H. (2015). Rainfall Runoff Analysis Using the Artificial Neural Network. Indian Journal of Science and Technology, 8(14), 1-7.

[13] R. Ashaary, N. A., Wan Ishak, W. H., \& Ku-Mahamud, K. R. (2015). Neural network application in the change of reservoir water level stage forecasting. Indian Journal of Science and Technology, 8(13), 1-6.

[14] Delgado, G., Aranda, V., Martínez, J. C., SánchezMarañón, M., Serrano, J. M., Sánchez, D., \& Vila, M. A. (2008). Building a fuzzy logic information network and a decision-support system for olive cultivation in Andalusia. Spanish Journal of Agricultural Research, (2), 252-263.

[15] Hepting, D. H., Maciag, T., \& Hill, H. (2012, January). Web-based support of crop selection for climate adaptation. In 2012 45th Hawaii International Conference on System Sciences (pp. 1227-1236). IEEE.

[16] Solanki, A. V. (2014). Data mining techniques using WEKA classification for sickle cell disease. International Journal of Computer Science and Information Technology, 5(4), 5857-5860.

[17] Uno, Y., Prasher, S. O., Lacroix, R., Goel, P. K., Karimi, Y., Viau, A., \& Patel, R. M. (2005). Artificial neural networks to predict corn yield from compact airborne spectrographic imager data. Computers and electronics in agriculture, 47(2), 149-161.

[18] Suraparaju, V., Mishra, B. \& Singh, Cd. (2011). Soybean Productivity Modelling using Decision Tree Algorithms. International Journal of Computer Applications. 27. 9758887. 10.5120/3314-4549.

[19] Wilcox, A., Perry, N. H., Boatman, N. D., \& Chaney, K. (2000). Factors affecting the yield of winter cereals in crop margins. The Journal of Agricultural Science, 135(4), 335346.

[20] Machine Learning in Agriculture: Applications and Techniques". https://medium.com/sciforce/machinelearning-in-agricultureapplications-and-techniques6ab501f4d1b5/

[21] Babu, S. (2013, August). A software model for precision agriculture for small and marginal farmers. In 2013 IEEE Global Humanitarian Technology Conference: South Asia Satellite (GHTC-SAS) (pp. 352-355). IEEE.
[22] Savla, A., Israni, N., Dhawan, P., Mandholia, A., Bhadada, H., \& Bhardwaj, S. (2015, March). Survey of classification algorithms for formulating yield prediction accuracy in precision agriculture. In 2015 International Conference on Innovations in Information, Embedded and Communication Systems (ICIIECS) (pp. 1-7). IEEE.

[23] Nasirahmadi, A., \& Behroozi-Khazaei, N. (2013). Identification of bean varieties according to color features using artificial neural network. Spanish Journal of Agricultural Research, 11(3), 670-677.

[24] Manjula, A., \& Narsimha, G. (2015, January). XCYPF: a flexible and extensible framework for agricultural crop yield prediction. In 2015 IEEE 9th International Conference on Intelligent Systems and Control (ISCO) (pp. 1-5). IEEE.

[25] Dahikar, S. S., \& Rode, S. V. (2014). Agricultural crop yield prediction using artificial neural network approach. International journal of innovative research in electrical, electronics, instrumentation and control engineering, 2(1), 683-686.

[26] Schuster, E. W., Kumar, S., Sarma, S. E., Willers, J. L., \& Milliken, G. A. (2011, November). Infrastructure for datadriven agriculture: identifying management zones for cotton using statistical modeling and machine learning techniques. In 2011 8th International Conference \& Expo on Emerging Technologies for a Smarter World (pp. 1-6). IEEE. 